\title{
gु
}

\section{Quantum criticality and the Tomonaga-Luttinger liquid in one-dimensional Bose gases}

\author{
Bing Yang, ${ }^{1,3}$ Yang-Yang Chen, ${ }^{2}$ Yong-Guang Zheng, ${ }^{1,3}$ Hui Sun, ${ }^{1,3}$ Han-Ning Dai, ${ }^{1,3}$ \\ Xi-Wen Guan, ${ }^{2,4, *}$ Zhen-Sheng Yuan, ${ }^{1,3,5,6, \uparrow}$ and Jian-Wei Pan ${ }^{1,3,5,6, *}$ \\ ${ }^{1}$ Hefei National Laboratory for Physical Sciences at Microscale and Department of Modern Physics, \\ University of Science and Technology of China, Hefei, Anhui 230026, China \\ ${ }^{2}$ State Key Laboratory of Magnetic Resonance and Atomic and Molecular Physics, Wuhan Institute of Physics and Mathematics, \\ Chinese Academy of Sciences, Wuhan 430071, China \\ ${ }^{3}$ Physikalisches Institut, Ruprecht-Karls-Universität Heidelberg, Im Neuenheimer Feld 226, 69120 Heidelberg, Germany \\ ${ }^{4}$ Department of Theoretical Physics, Research School of Physics and Engineering, Australian National University, \\ Canberra ACT 0200, Australia \\ ${ }^{5}$ CAS-Alibaba Quantum Computing Laboratory, Shanghai 201315, China \\ ${ }^{6}$ CAS Centre for Excellence and Synergetic Innovation Centre in Quantum Information and Quantum Physics, \\ University of Science and Technology of China, Hefei, Anhui 230026, China \\ (Received 26 December 2016; revised manuscript received 18 May 2017; published 18 October 2017)
}

\begin{abstract}
We experimentally investigate the quantum criticality and Tomonaga-Luttinger liquid (TLL) behavior within one-dimensional (1D) ultracold atomic gases. Based on the measured density profiles at different temperatures, the universal scaling laws of thermodynamic quantities are observed. The quantum critical regime and the relevant crossover temperatures are determined through the double-peak structure of the specific heat. In the TLL regime, we obtain the Luttinger parameter by probing sound propagation. Furthermore, a characteristic power-law behavior emerges in the measured momentum distributions of the 1D ultracold gas, confirming the existence of the TLL.
\end{abstract}

DOI: 10.1103/PhysRevLett.119.165701

Quantum many-body systems can exhibit phase transitions even at zero temperature [1,2]. Here, quantum fluctuations arising from Heisenberg's uncertainty relation drive the transition from one phase to another. In this regard, onedimensional (1D) quantum systems are special owing to the significant microscopic fluctuations that induce a continuous phase transition between a disordered state and a Tomonaga-Luttinger liquid (TLL) [3-7]. Near the transition point, a quantum critical (QC) regime emerges at finite temperatures and separates these two phases [1,2,8]. Although the 1D low-energy physics is generally described by the well-established TLL theory [3], experimental investigations of the TLL and its related quantum criticality are rare [9-11]. In this context, signatures of TLL were found in some 1D systems, such as organic conductors [12], carbon nanotubes [13], spin ladders [10], and quantum gases [14,15]. Among these strongly correlated systems, ultracold atomic gases offer a great precision and tunability for studying quantum phase transitions $[16,17]$ and critical phenomena [18,19]. However, observation of quantum criticality and determination of the TLL boundary in 1D quantum gases remains elusive.

In this Letter, we report the observation of quantum criticality and evidence of TLL in 1D ultracold Bose gases of ${ }^{87} \mathrm{Rb}$. The atomic samples at different temperatures are prepared in well-designed 1D harmonic potentials. Using a high-resolution microscope, we measure the density profiles by in situ absorption imaging. The density scaling law is obtained by rescaling these measurements at different temperatures and chemical potentials. Based on the thermodynamic relations [20-22], we derive the pressures and entropy densities, which exhibit similar universal scaling around the critical point. Moreover, we determine two crossover branches that distinguish the QC regime from the classical gas (CG) and the TLL through the double-peak structure of the specific heat. To further investigate the degenerate gas, we probe the propagations of density disturbances and acquire the Luttinger parameters. Then we characterize the phase correlation of the $1 \mathrm{D}$ ultracold gas through its momentum distribution. According to the bosonization-based theory [3,23], the obtained power-law behavior in the momentum profiles confirms the existence of the TLL.

The experiment starts by adiabatically loading a BoseEinstein condensate of $\sim 2 \times 10^{5}$ atoms into a single layer of a pancake-shaped trap. We then confine the atoms into an array of isolated tube-shaped traps arranged in a plane by superimposing another red-detuned lattice with wavelength $\lambda_{r}=1534 \mathrm{~nm}$ into the system [see Fig. 1(a)]. Owing to the homogeneity of the light beams among these tubes, they are identical to each other with trap frequencies $\omega_{x}=$ $2 \pi \times 22.2(1) \mathrm{Hz}$ and $\omega_{\perp}=\sqrt{\omega_{y} \omega_{z}}=2 \pi \times 7.99(1) \mathrm{kHz}$. The spatial resolution of the imaging system $(1.0 \mu \mathrm{m})$ is slightly larger than the lattice spacing $\lambda_{r} / 2=767 \mathrm{~nm}$. After acquiring around 400 high-resolution images for each experimental setting, we then obtained very precise 1D density profiles by averaging these images. The measured temperatures [24], $T=18-74 \mathrm{nK}$, and corresponding 
(a)
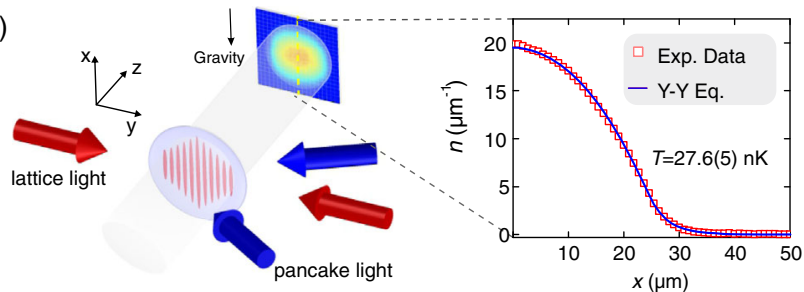

(b)
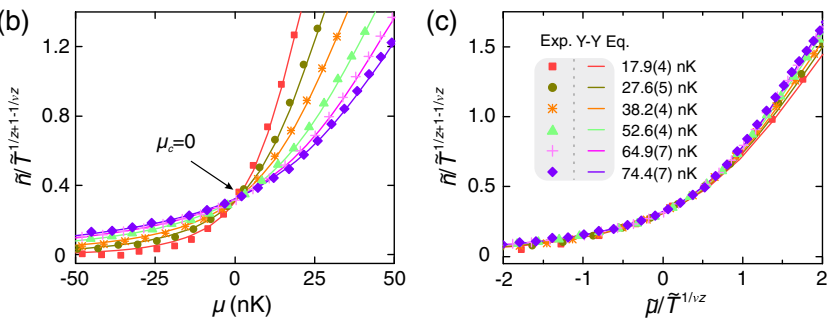

FIG. 1. Experimental setup and density scaling law. (a) The 1D system consists of an array of tubes created by a blue-detuned "pancake" lattice and a red-detuned retro-reflected lattice. The density profiles are measured by in situ absorption imaging. The inset shows an average line density in comparison with the prediction of the Yang-Yang (YY) equation. (b) The rescaled densities at different temperatures intersect at the critical point $\mu_{c}=0$. Here $\tilde{n}=n / c, \quad \tilde{\mu}=\mu /\left(\hbar^{2} c^{2} / 2 m\right)$, and $\tilde{T}=k_{B} T /$ $\left(\hbar^{2} c^{2} / 2 m\right)$, with $c=-2 / a_{1 \mathrm{D}}$. The symbols denote the experimental data; solid curves stand for the theoretical predictions. (c) At different temperatures, the rescaled densities against $\tilde{\mu} / \tilde{T}^{1 / \nu z}$ collapse into a single curve around $\mu_{c}$.

chemical potentials in the trap center, $\mu_{0}=67-93 \mathrm{nK}$, satisfy the $1 \mathrm{D}$ conditions of $k_{B} T, \mu_{0} \ll \hbar \omega_{\perp}$. The minimal entropy per particle of 0.055(1) $k_{B}$ at $T=17.9(4) \mathrm{nK}$ and $\mu_{0}=67.1(1) \mathrm{nK}$ indicates that the $1 \mathrm{D}$ gas is strongly degenerate. The dimensionless interaction parameter $\gamma \approx$ $2 m a_{1 \mathrm{D}} \omega_{\perp} /\left(\hbar n_{0}\right)=0.04 \ll 1$ suggests that the central region of the system is in the weakly interacting regime, where $m$ is the mass of the atom, $a_{1 \mathrm{D}}$ is the $1 \mathrm{D}$ effective scattering length, and $n_{0}$ is the line density at the center of the 1D tube. Under such experimental conditions, the 1D Bose gas can be described by the Lieb-Liniger model [28]. Within the local density approximation (LDA), the measured densities agree well with the theoretical predictions from the YY exact grand canonical theory [21] [see Fig. 1(a)].

For the one-dimensional Lieb-Liniger model $[22,28]$ at zero temperature, a vacuum-to-TLL phase transition occurs when we change the chemical potential in a positive direction across the critical point $\mu_{c}=0$. At finite temperatures, a QC regime emerges near $\mu_{c}$ and separates the CG and the TLL phase. In the QC regime, the correlation length $\xi$ diverges as $\xi \propto\left|\mu-\mu_{c}\right|^{-\nu}$, and the energy gap $\Delta$ is inversely proportional to the correlation length $\Delta \propto \xi^{-z} \propto\left|\mu-\mu_{c}\right|^{\nu z}$, which vanishes as $\mu \rightarrow \mu_{c}[1,2,22]$. Here $\nu$ and $z$ are defined as the correlation length exponent and the dynamic critical exponent, respectively. In this context, the particle density in QC obeys a universal scaling law, as $n(\mu, T)=T^{(d / z)+1-(1 / \nu z)} \mathcal{F}\left[\left(\mu-\mu_{c}\right) / T^{(1 / \nu z)}\right]$, where the dimensionality is $d=1$ and $\mathcal{F}(x)$ is the scaling function [5].

Such a universal scaling law is extracted from the density profiles at temperatures ranging from 17.9(4) $\mathrm{nK}$ to 74.4(7) $\mathrm{nK}$. As shown in Fig. 1(b), we identify the critical point using that the scaled density becomes temperature independent at $\mu_{c}$, i.e., the density profiles at different temperatures intersect at the critical point. The critical exponents $\nu$ and $z$ are determined by the overlapping feature of the rescaled density profiles [24]. The rescaled measurements fall into a single curve with $\nu=0.56_{-0.08}^{+0.07}$ and $z=2.3_{-0.3}^{+0.6}$ [Fig. 1(c)], confirming the emergence of the quantum critical scaling. Here the uncertainties correspond to a $95 \%$ confidence level. The critical exponents agree with the predictions from the YY equation, $\nu=0.5$ and $z=2$ [21,22]. The above properties of densities at various temperatures and chemical potentials reveal the nature of scaling invariance.

The thermodynamics of the 1D system at equilibrium are described by the EOS. We can derive the local pressure EOS from the atomic density via $p(\mu, T)=$ $\int_{-\infty}^{\mu} n\left(\mu^{\prime}, T\right) d \mu^{\prime}$ [20] by introducing a proper cutoff in the CG regime, as shown in Fig. 2(a). For the lowest temperature experimentally probed $T=17.9(4) \mathrm{nK}$, the population below $\mu_{c}$ is negligible and therefore the pressure approaches that of the zero temperature result. Whereas at higher temperatures, the pressure curves split clearly in the QC regime and bunch up again at large chemical potentials. From the pressure EOS, one can obtain other thermodynamic properties. For example, the entropy density can be deduced as $S(\mu, T)=[\partial p(\mu, T) / \partial T]_{\mu}$ [20]. Figure 2(b) shows the entropy densities extracted from experimental data and the theoretical curves. Peaks arise in the entropy density curves and become flatter at higher temperatures, revealing enhanced disorder in the QC regime. Moreover, both the pressure and entropy densities [Figs. 2(c) and 2(d)] have similar universal scaling laws with the same critical exponents as those in the density scaling function [24].

In the scenario of quantum criticality, determining the crossover temperatures $T^{*}$ in quantum gases poses theoretical [6,7] and experimental challenges [19]. Here we make a distinction of different regimes through the feature of specific heat, $c_{V}=T\left(\partial^{2} p / \partial T^{2}\right)_{\mu}$, which manifests different scales of the energy fluctuations in the grand canonical ensemble. In ultracold atomic gases, obtaining a $c_{V}$ of merit requires high-precision density measurements. At finite temperatures, a double-peak structure of the specific heat appears and marks two crossover temperatures fanning out from the critical point [Fig. 3(a)]. The peak values of the $c_{V}$ constitute two branches of the QC crossover boundaries in Fig. 3(b). Here, the theoretical curves and contour plot of specific heat are numerically 

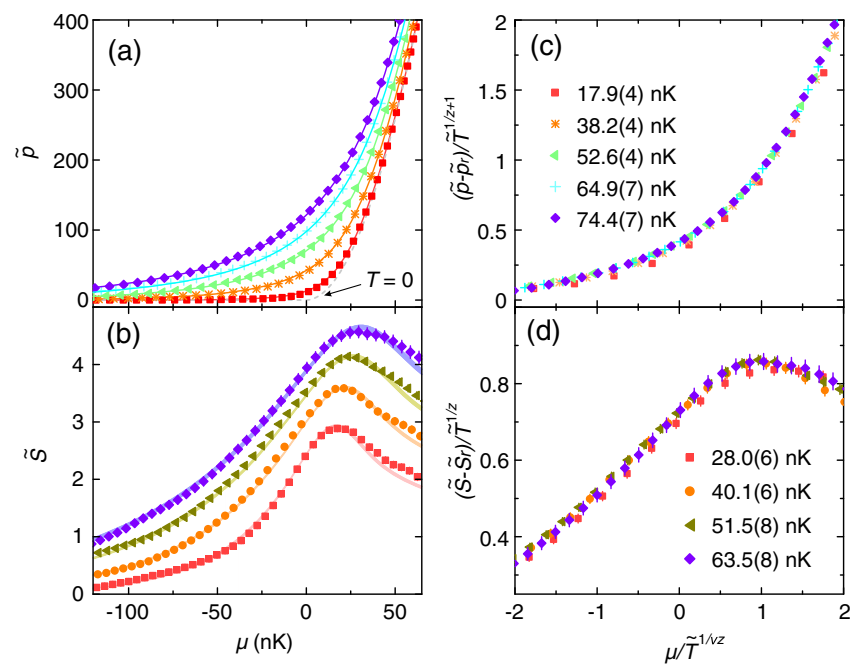

FIG. 2. The dimensionless pressure $\tilde{p}=p /\left[\hbar^{2} c^{3} /(2 m)\right]$ and entropy density $\tilde{S}=S /\left(k_{B} c\right)$. The solid lines and the shaded curves are the theoretical predictions from the YY equation. The thickness of the shaded area indicates errors arising from the temperature uncertainties. (a) The pressure equation of state (EOS) (symbols) at different temperatures is deduced from the density profiles. (b) Symbols denote the experimental data of entropy densities extracted from the pressure. [(c) and (d)] Using the same critical exponents $\nu$ and $z$ for the density, the rescaled pressures and entropy densities overlap and collapse into a single curve around the critical point. Here $p_{r}$ and $S_{r}$ are the regular parts of the scaling functions. The error bars denote the $\pm 1 \sigma$ statistical errors.

calculated via the second-order derivation of the pressure. The left branch indicates the 1D degenerate condition, i.e., the thermal de Broglie wavelength $\lambda_{T}=\sqrt{2 \pi \hbar^{2} /\left(m k_{B} T\right)}$ is approximate to the atomic spacing $1 / n$. The right branch separates the QC and a linear-dispersion TLL regime with a crossover temperature $T^{*} \sim\left|\mu-\mu_{c}\right|^{\nu z}$. In the TLL regime of the phase diagram, the specific heat at a certain chemical potential depends almost linearly on temperature [24], reflecting a collective behavior of the quantum liquid. The peaks of $S$ and the valleys of $c_{V}$ reveal that quantum fluctuations dominate the quantum critical behavior.

The low-energy properties of the TLL can be fully described by the sound velocity $v_{s}$ and Luttinger parameter $K[4,6,29]$. Here $v_{s}$ represents the propagating velocity of density disturbances, which satisfies a linear dispersion relation $\omega=v_{s}|k|$. Experimentally, the sound velocity is obtained by monitoring the propagation of density perturbations in the 1D tubes. We apply a magnetic gradient along the longitudinal direction ( $x$ axis) to create a spatially dependent Zeeman splitting, which enables a spatially resolved transfer of atoms from $\left|F=1, m_{F}=-1\right\rangle$ into $\left|F=2, m_{F}=0\right\rangle$ sublevel via microwave (MW) transitions. With a resonant light pulse to remove the atoms in the $|F=2\rangle$ states, density dips are generated in the center of the 1D tubes. The profile of these defects is
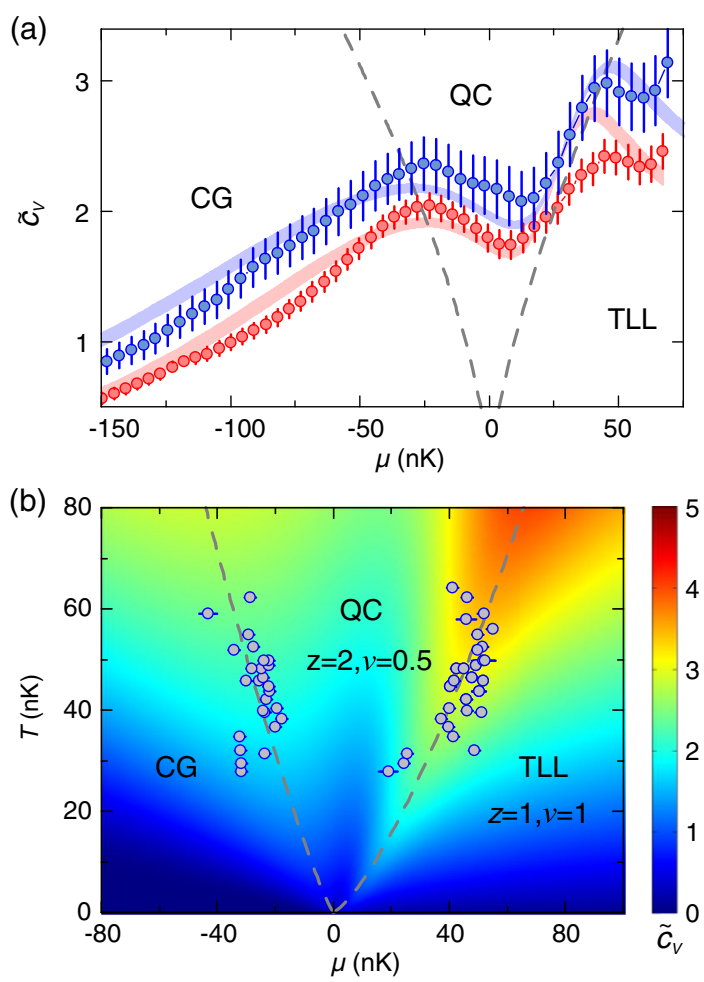

FIG. 3. The dimensionless specific heat $\tilde{c}_{V}=c_{V} /\left(k_{B} c\right)$ and its phase diagram. (a) Experimental specific heat (red and blue circles) at the temperatures of $T=40(1) \mathrm{nK}$ and $T=50(1) \mathrm{nK}$, respectively. The double-peak feature of specific heat marks out the regimes CG, QC, and TLL. The shaded areas indicate the theoretical predictions by taking account of the temperature uncertainty. (b) Contour plot of specific heat in the $T-\mu$ plane in which its peaks (dashed lines) separate three fluctuation regimes. The QC and the TLL are classified by different critical exponents $z$ and $\nu$. The dots denote the experimental data of the specific heat peaks that mark the two crossover temperatures $T^{*}$. Here the error bars denote the $\pm 1 \sigma$ fitting errors.

approximately Gaussian $\eta n_{0} e^{-x^{2} / 2 w^{2}}$, where the relative amplitude $\eta$ and the width $w$ are tailored by adjusting the MW strength. As shown in the insets of Fig. 4(a), such negative perturbations split into two parts and then symmetrically propagate along the $1 \mathrm{D}$ tubes. For different perturbing amplitudes, we resolve a linear relation between the $v_{s}$ and the square root of remaining density $\sqrt{n_{0}(1-\eta / 2)}$, as $v_{s}(\eta)=v_{s}(0) \sqrt{1-\eta / 2}[30-32]$. Based on this relation, the sound velocities at vanishing perturbations are determined as 2.24(1) and 2.21(1) mm/s for $T=40(3) \mathrm{nK}$ and $T=50(3) \mathrm{nK}$, respectively. For a uniform quantum gas, the Luttinger parameter $K$ and the $v_{s}$ have a relation as $K=\hbar \pi n / m v_{s}$ [24,33]. Whereas for TLL in the harmonic trap, we can get an averaged Luttinger parameter $\bar{K}$ by employing the averaged density and sound velocity over the TLL regime. In the cases of $T=40(3) \mathrm{nK}$ and $T=50(3) \mathrm{nK}$, the $\bar{K}$ acquired from the measured sound velocities and atomic densities are 16.9 and 17.2 , respectively [24]. 
(a)

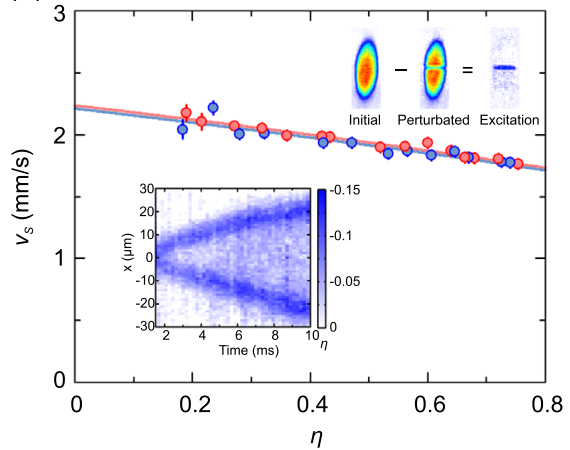

(b)

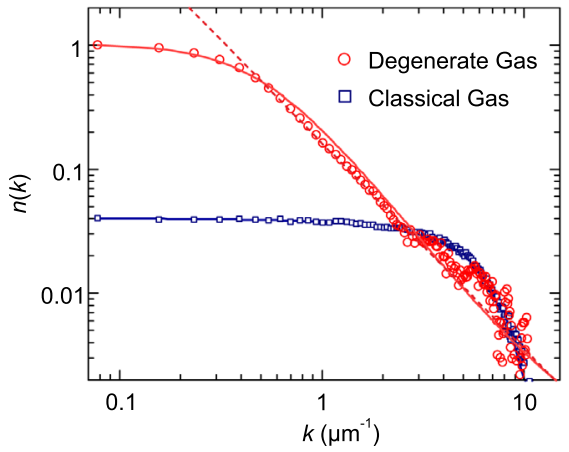

(c)

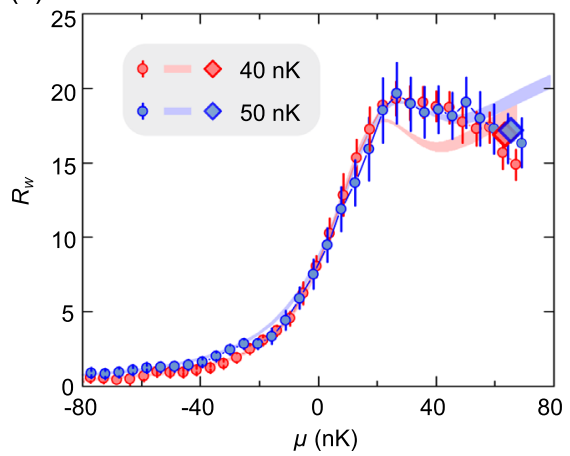

FIG. 4. Evidences for TLL. (a) Measurements of sound velocity. The upper inset shows the generation process of the excitation in the sample. The excitation signal is obtained by subtracting the perturbated cloud from the initial density. The lower inset shows the propagation of a negative perturbation in 1D tubes with amplitude $\eta=0.17(1)$ and a Gaussian width $w=4.0(4) \mu \mathrm{m}$. The red and blue circles are experimental sound velocities versus excitation ratios at temperature $T=40(3) \mathrm{nK}$ and $T=50(3) \mathrm{nK}$. The red and blue curves are the fitting results. (b) Momentum profiles of the 1D gases. The red circles and blue squares stand for the experimental momentum distributions for a degenerate gas at $T=40(1) \mathrm{nK}$ and a classical gas at $T=209(1) \mathrm{nK}$, respectively. All the experimental data are normalized to the zero-momentum value of the degenerate gas. The red solid curve is the theoretical prediction by considering the finite-temperature effect and the averaged Luttinger parameter. The red dashed curve is an auxiliary straight line with a slope of -1.66, while the blue solid curve follows a Gaussian distribution. (c) Wilson ratio $R_{W}$ and Luttinger parameter $K$. The red and blue circles are experimental $R_{W}$ at $T=40(1) \mathrm{nK}$ and $T=50(1) \mathrm{nK}$, while the shaded areas indicate the theoretical predictions. Two diamond points represent the averaged Luttinger parameter of the TLL regime. The error bars represent the $\pm 1 \sigma$ statistical errors.

For the TLL, one characteristic property of the TLL is the interaction-dependent power-law behavior of correlation functions $[3,4,23,29]$. Such quasilong-range order is evident in the first-order correlation function $g^{(1)}(x)=$ $\left\langle\psi^{\dagger}(x) \psi(0)\right\rangle$, which features a power-law decay $g^{(1)}(x) \propto$ $x^{-1 / 2 K}$ in the uniform system at zero temperature [3]. The momentum distribution of TLL is the Fourier transform of this correlation function, i.e., $n(k)=\int_{-\infty}^{\infty} d x e^{-i k x} g^{(1)}(x)$. In a low-temperature atomic gas, the dominant phase fluctuations give rise to a finite correlation length $l_{\phi}=\hbar v_{s} K /\left(\pi k_{B} T\right)$ [17,23] and modify the power-law behavior of the momentum distribution. In this case, $n(k) \simeq$ $A(K) \operatorname{Re}\left[\Gamma\left(1 / 4 K+i k l_{\phi} / 2 K\right) / \Gamma\left(1-1 / 4 K+i k l_{\phi} / 2 K\right)\right]$, where $A(K)$ is a $K$-dependent parameter [23]. If the system size $L$ is much larger than the phase correlation length $l_{\phi}$, the inhomogeneity of the harmonic trap can be safely neglected and the system can be treated using the LDA $[23,34,35]$.

To access the momentum distribution of the 1D gases, we utilize a focusing technique during the time of flight [36] instead of a conventional long-time expansion [24]. The momentum distribution of a $1 \mathrm{D}$ gas at $T=40(1) \mathrm{nK}$ is displayed by a log-log plot in Fig. 4(b). For this nonuniform system, the averaged Luttinger parameter of the TLL regime $\left(\mu \geq T^{*}\right)$ is $\bar{K}=15.9$, indicating a correlation length of $l_{\phi}=1.9 \mu \mathrm{m}$. Thermal fluctuations break the long-range phase correlations, making the momentum distribution for $k<1 / l_{\phi}$ rather flat. Another characteristic length is the healing length $\sim 0.1 \mu \mathrm{m}$, which determines the high-momentum cutoff of our measurements. The measured $n(k)$ exhibits a power-law decay at intermediate momenta with a linear slope of $-1.66\left(1 / l_{\phi} \leq k \leq 20 / l_{\phi}\right)$. As the system satisfies the condition of LDA $\left(L \gg l_{\phi}\right)$, we obtain a theoretical curve of $n(k)$ by using the parameter $\bar{K}$. This curve has an asymptotic power-law decay with the slope $-1+1 / 2 K$ at large momenta $\left(k>40 / l_{\phi}\right)[23,24]$. The inhomogeneity of the harmonic potential might lead to some modification to $n(k)$, which would have an extended flat region at small momenta and a Lorentzian distribution with power-law exponent -2 at intermediate momenta [34,35]. However, within the accessible range, our experimental result agrees well with the theoretical prediction [23], indicating that TLL behavior dominates the system and the momentum distribution can be qualitatively understood by considering a uniform gas with the same $\bar{K}$. For a comparison, we also measure the momentum distribution of a classical gas with $T=209(1) \mathrm{nK}$ and $\mu_{0}=-111(1) \mathrm{nK}$. Both the spatial and the momentum distribution of this gas show classical Gaussian profiles as predicted by the Boltzmann distributions.

In the TLL regime, although the Fermi liquid theory cannot describe 1D systems due to collective behavior herein, two important features of quantum liquid still retain; i.e., the compressibility $\kappa$ is independent of temperature and the specific heat $c_{V}$ is linearly proportional to temperature [37-39]. Therefore, we employ a dimensionless Wilson ratio to characterize different regimes, $R_{W}=$ $\left(\pi^{2} k_{B}^{2} / 3\right) \kappa /\left(c_{V} / T\right)[37,38,40,41]$. An equivalence between the Wilson ratio and the Luttinger parameter has been proved in the uniform TLLs $[38,41]$. This relation indicates that the particle number fluctuation and the energy fluctuation are on an equal footing with respect to $T$. In our 
experiment, the derived $R_{W}$ approaches the averaged $K$ in the TLL regime [see Fig. 4(c)]. The connection between the $R_{W}$ and $K$ provides a novel method for determining the Luttinger parameter in the solid-state system [37], where the sound velocity is hard to measure. Meanwhile, the crossover features of the QC regime can also be characterized by the "critical cone" in the phase diagram of $R_{W}$ [24].

In summary, we present a systematic study of the quantum criticality and TLL behavior in 1D quantum gases. The 1D density profiles of ultracold Bose gases with a minimum entropy per particle $0.055 k_{B}$ have been obtained with a high precision. Using these density profiles, we have determined universal scaling laws, the EOS, and crossover temperatures of this system. Afterwards, the Luttinger parameters have been obtained by the measured sound velocities and atomic densities. In our nonuniform system, the momentum distribution that exhibits a power-law decay at intermediate momenta is well consistent with the TLL theory. Our experiment provides prototypical methods for studying quantum critical phenomena and quantum liquids, not only in other spinless quantum gases $[14,15,33,42,43]$ but also in quantum manybody systems involving rich spin (and charge) interactions and symmetries, such as spin chains $[11,44]$, the YangGaudin model [45], and the Hubbard model [46].

The authors thank H. Zhai, Q. Zhou, A. del Campo, S. Jochim, S. Whitlock, Y.-J. Deng, Z.-C. Yan, A. Truscott, and T. Giamarchi for helpful discussions. This work has been supported by the National Key R\&D Program of China (Grants No. 2016YFA0301600 and No. 2017YFA0304500), the NNSFC (Grants No. 11534014, No. 11374331, and No. 91221204), and the Chinese Academy of Sciences. X.W.G. has been partially supported by the Australian Research Council.

B. Y. and Y.-Y. C. contributed equally to this work.

*xiwen.guan@anu.edu.au

†yuanzs@ustc.edu.cn

\#pan@ustc.edu.cn

[1] S. Sachdev, Quantum Phase Transitions (Wiley Online Library, New York, 2007).

[2] M. P. A. Fisher, P. B. Weichman, G. Grinstein, and D. S. Fisher, Phys. Rev. B 40, 546 (1989).

[3] F. D. M. Haldane, J. Phys. C 14, 2585 (1981).

[4] T. Giamarchi, Quantum Physics in One Dimension (Oxford University Press, Oxford, 2004).

[5] Q. Zhou and T.-L. Ho, Phys. Rev. Lett. 105, 245702 (2010).

[6] M. A. Cazalilla, R. Citro, T. Giamarchi, E. Orignac, and M. Rigol, Rev. Mod. Phys. 83, 1405 (2011).

[7] X.-W. Guan, M. T. Batchelor, and C. Lee, Rev. Mod. Phys. 85, 1633 (2013).

[8] S. Sachdev and B. Keimer, Phys. Today 64, No. 2, 29 (2011).
[9] B. Lake, D. A. Tennant, C. D. Frost, and S. E. Nagler, Nat. Mater. 4, 329 (2005).

[10] C. Rüegg, K. Kiefer, B. Thielemann, D. F. McMorrow et al., Phys. Rev. Lett. 101, 247202 (2008).

[11] Y. Kono, T. Sakakibara, C. P. Aoyama, C. Hotta, M. M. Turnbull, C. P. Landee, and Y.Takano, Phys. Rev. Lett. 114, 037202 (2015).

[12] A. Schwartz, M. Dressel, G. Grüner, V. Vescoli, L. Degiorgi, and T. Giamarchi, Phys. Rev. B 58, 1261 (1998).

[13] Z. Yao, H. W. C. Postma, L. Balents, and C. Dekker, Nature (London) 402, 273 (1999).

[14] B. Paredes, A. Widera, V. Murg, O. Mandel, S. Fölling, I. Cirac, G. V. Shlyapnikov, T. W. Hänsch, and I. Bloch, Nature (London) 429, 277 (2004).

[15] S. Hofferberth, I. Lesanovsky, T. Schumm, A. Imambekov, V. Gritsev, E. Demler, and J. Schmiedmayer, Nat. Phys. 4, 489 (2008).

[16] M. Greiner, O. Mandel, T. Esslinger, T. W. Hänsch, and Immanuel Bloch, Nature (London) 415, 39 (2002).

[17] I. Bloch, J. Dalibard, and W. Zwerger, Rev. Mod. Phys. 80, 885 (2008).

[18] T. Donner, S. Ritter, T. Bourdel, A. Ottl, M. Kohl, and T. Esslinger, Science 315, 1556 (2007).

[19] X. Zhang, C.-L. Hung, S.-K. Tung, and C. Chin, Science 335, 1070 (2012).

[20] T.-L. Ho and Q. Zhou, Nat. Phys. 6, 131 (2010).

[21] C. N. Yang and C.P. Yang, J. Math. Phys. 10, 1115 (1969).

[22] X. W. Guan and M. T. Batchelor, J. Phys. A 44, 102001 (2011)

[23] M. A. Cazalilla, J. Phys. B 37, S1 (2004).

[24] See Supplemental Material http://link.aps.org/supplemental/ 10.1103/PhysRevLett.119.165701 for the details of the experimental method and relevant theory, which includes Refs. [25-27].

[25] T. Yefsah, R. Desbuquois, L. Chomaz, K. J. Günter, and J. Dalibard, Phys. Rev. Lett. 107, 130401 (2011).

[26] G. Reinaudi, T. Lahaye, Z. Wang, and D. Guéry-Odelin, Opt. Lett. 32, 3143 (2007).

[27] H.-N. Dai, B. Yang, A. Reingruber, X.-F. Xu, X. Jiang, Y.-A. Chen, Z.-S. Yuan, and J.-W. Pan, Nat. Phys. 12, 783 (2016).

[28] E. H. Lieb and W. Liniger, Phys. Rev. 130, 1605 (1963).

[29] F. D. M. Haldane, Phys. Rev. Lett. 47, 1840 (1981).

[30] M. R. Andrews, D. M. Kurn, H. J. Miesner, D. S. Durfee, C. G. Townsend, S. Inouye, and W. Ketterle, Phys. Rev. Lett. 79, 553 (1997).

[31] G. M. Kavoulakis and C. J. Pethick, Phys. Rev. A 58, 1563 (1998).

[32] R. Meppelink, S. B. Koller, and P. van der Straten, Phys. Rev. A 80, 043605 (2009).

[33] E. Haller, R. Hart, M. J. Mark, J. G. Danzl, L. Reichsöllner, M. Gustavsson, M. Dalmonte, G. Pupillo, and H.-C. Nägerl, Nature (London) 466, 597 (2010).

[34] S. Richard, F. Gerbier, J. H. Thywissen, M. Hugbart, P. Bouyer, and A. Aspect, Phys. Rev. Lett. 91, 010405 (2003).

[35] T. Jacqmin, B. Fang, T. Berrada, T. Roscilde, and I. Bouchoule, Phys. Rev. A 86, 043626 (2012).

[36] I. Shvarchuck, C. Buggle, D. S. Petrov, K. Dieckmann, M. Zielonkovski, M. Kemmann, T. G. Tiecke, W. von Klitzing, 
G. V. Shlyapnikov, and J. T. M. Walraven, Phys. Rev. Lett. 89, 270404 (2002).

[37] K. Ninios, T. Hong, T. Manabe, C. Hotta, S. N. Herringer, M. M. Turnbull, C. P. Landee, Y. Takano, and H. B. Chan, Phys. Rev. Lett. 108, 097201 (2012).

[38] X. W. Guan, X. G. Yin, A. Foerster, M. T. Batchelor, C. H. Lee, and H. Q. Lin, Phys. Rev. Lett. 111, 130401 (2013).

[39] A. J. Schofield, Contemp. Phys. 40, 95 (1999).

[40] K. G. Wilson, Rev. Mod. Phys. 47, 773 (1975).

[41] Y.-C. Yu, Y.-Y. Chen, H.-Q. Lin, R. A. Römer, and X. W. Guan, Phys. Rev. B 94, 195129 (2016).
[42] T. Kinoshita, T. Wenger, and D. S. Weiss, Science 305, 1125 (2004).

[43] T. Jacqmin, J. Armijo, T. Berrada, K. V. Kheruntsyan, and I. Bouchoule, Phys. Rev. Lett. 106, 230405 (2011).

[44] G. Pagano, M. Mancini, G. Cappellini, P. Lombardi et al., Nat. Phys. 10, 198 (2014).

[45] Y.-an Liao, A. S. C. Rittner, T. Paprotta, W. Li, G. B. Partridge, R. G. Hulet, S. K. Baur, and E. J. Mueller, Nature (London) 467, 567 (2010).

[46] F. H. Essler, H. Frahm, F. Göhmann, A. Klümper, and V. E. Korepin, The One-Dimensional Hubbard Model (Cambridge University Press, Cambridge, 2005). 